\title{
Barcelona
}

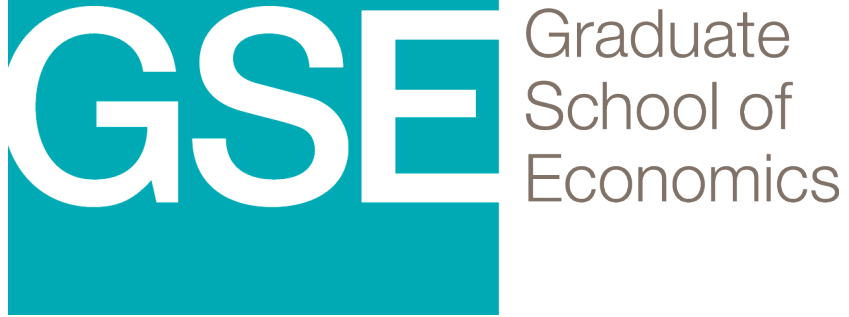

\section{An Experimental Study of Persuasion Bias and Social Influence in Networks}

\author{
Jordi Brandts \\ Ayça Ebru Giritligil \\ Roberto A. Weber
}

May 2015

Barcelona GSE Working Paper Series

Working Paper $n^{\circ} 829$ 


\title{
An Experimental Study of Persuasion Bias and Social
}

\section{Influence in Networks}

\author{
Jordi Brandts $^{*}$, Ayça Ebru Giritligil ${ }^{* *}$ and Roberto A. Weber ${ }^{* * *}$
}

May 22, 2015

\begin{abstract}
In many areas of social life, individuals receive information about a particular issue of interest from multiple sources. When these sources are connected through a network, then proper aggregation of this information by an individual involves taking into account the structure of this network. The inability to aggregate properly may lead to various types of distortions. In our experiment, four agents all want to find out the value of a particular parameter unknown to all. Agents receive private signals about the parameter and can communicate their estimates of the parameter repeatedly through a network, the structure of which is known by all players. We present results from experiments with three different networks. We find that the information of agents who have more outgoing links in a network gets more weight in the information aggregation of the other agents than under optimal updating. Our results are consistent with the model of "persuasion bias" of DeMarzo et al. (2003).
\end{abstract}

Keywords: Persuasion Bias, Experiments, Bounded Rationality

JEL Classification Codes: C92, D03, D83

\footnotetext{
Corresponding autor. Instituto de Analisis Economico (CSIC) and Barcelona GSE. Address: Campus UAB, 08193 Bellaterra (Barcelona), Spain. e-mail: jordi.brandts@iae.csic.es, 34-935806612

** Murat Sertel Center for Advanced Economic Studies, İstanbul Bilgi University, Santral Campus L1-103, Kazım Karabekir Cad. 2/13, Eyüp 34060 İstanbul, Turkey. e-mail: ayca.ebru@bilgi.edu.tr

**** Department of Economics, University of Zurich, Blümlisalpstrasse 10, CH-8006 Zurich, Switzerland.

email: roberto.weber@econ.uzh.ch
} 


\section{Introduction}

In many important social and economic situations, individuals receive information about a particular issue from multiple sources and also transmit information to multiple others. Examples are the sharing of political opinions among voters or of information about prospective job candidates in an organization's hiring process. When people exchange opinions about such issues within a group, some of the group members may have a stronger influence on the group's opinion than others due to the quality or accuracy of the information. However, influence may also often be due to social factors like the resources some people have available to invest in spreading their opinions, how well considered their opinions are in society or how well connected they are to others.

One basic source of social influence occurs in communication through networks, where there exists the possibility of one person's information reaching a particular other person repeatedly. When individuals are connected through a network, then they may receive information both directly and indirectly from the same source and send information directly and indirectly to a particular other source. In such situations, perfectly rational aggregation of information by an individual involves taking into account the structure of the network and adjusting how one weighs the information one receives accordingly. However, boundedly rational agents may have difficulties with this process and aggregate in biased ways-for example, by failing to adjust properly for repetitions of information. ${ }^{1}$ In this paper we present results from laboratory experiments that shed light on how people aggregate information when they are connected in a network.

DeMarzo et al. (2003) present a stylized version of an information aggregation situation in a network, together with a model of a particular bias to which boundedly rational

\footnotetext{
${ }^{1}$ See, for instance, Gale and Kariv (2003), Golub and Jackson (2010) and Acemoglu et al. (2011).
} 
agents may fall prey. They posit a situation in which a set of agents all want to find out the value of a numerical parameter. Each agent starts with some initial private information about the parameter, the aggregation of which is all the information available to the group of agents in the network. Agents then communicate their estimates about the true parameter to one another through the network. The network consists of a number of connections between the set of agents that specify who sends information to whom-or, alternatively, who listens to whom. There are multiple periods of communication between the agents, a feature meant to represent a lengthy deliberation process. In each period, each agent listens to the estimates of those who, following the network structure, send him information and sends his estimate to those who listen to him. After each period, each agent can update his own estimate in order to approximate the true parameter based on any new information received from other agents.

There is a rational way to aggregate information in such a setting, which involves agents discounting information that reaches them repeatedly through distinct channels in the network. But it is possible that boundedly rational individuals will not use this optimal process. A priori, there are many distinct ways in which information could be aggregated non-optimally. DeMarzo et al. (2003) propose a particular model of boundedly rational information aggregation, based on DeGroot (1974), which leads to "persuasion bias." According to this model, all agents treat all information they receive as new, ignoring the fact that an estimate received in a particular period may contain information that has already been received-directly or indirectly_from another source. Agents treat the information they receive in each period as new and independent and do not adjust for the fact that over time the information of some agents might contain more repetitions than that of others. The implications of this kind of biased information aggregation depend on the structure of the network. Some networks may cancel out the biased weighting of information, so that agents subject to the boundedly rational persuasion bias may nevertheless reach an unbiased 
estimate of the true parameter. However, other networks will not have this property and the consequence will be that the presence of a bias at the level of individuals processing information yields groups that arrive at biased estimates of the parameter.

The model of bias proposed by DeMarzo et al. (2003) is a plausible one. Nevertheless, it is not a priori obvious that if people turn out to be biased, they will be so in the precise way posited by the model. There are many ways to aggregate information and addressing the questions of which bias will occur and how it will affect group outcomes requires empirical information. Therefore, we use laboratory experiments to explore the internal validity of the persuasion bias model. The laboratory provides an ideal environment to study the relation between network structures and the kind of information and communication processes necessary to test the persuasion bias model. The two principal values of laboratory experiments are control and replicability. Causal knowledge requires controlled variation (Falk and Heckman, 2009) and the laboratory allows for tight control over the environment in which interaction takes place. At the same time, the laboratory allows the generation of sufficient data to test theoretical predictions in a simple way.

In this paper, we present results from experiments with three different networks. The first two are directly inspired by the discussion in DeMarzo et al. (2003). We study behavior using their examples of both "balanced" and "unbalanced" networks of four agents. In the balanced network, four agents are located in a circle. They all receive one piece of information and all send their estimate of the parameter to and receive it from the two agents closest to them. In such a balanced network, DeMarzo et al. (2003) predict no bias in the consensus estimates of the group. In the unbalanced network, the four agents all receive one piece of information, but some agents send information to a larger number of others and receive information from a smaller number of agents than others. In such an unbalanced 
network, DeMarzo et al.'s (2003) model predicts a precise biased outcome to which the group's estimates should converge.

We find that observed behavior is consistent with persuasion bias and, importantly, with many of the precise predictions of DeMarzo et al.'s (2003) model. In the balanced network, subjects' estimates move in the direction of estimates in which the private information of all four players carries identical weights, as predicted by the theory. In contrast, in the unbalanced network, subjects tend towards biased estimates that give greater weight to the private information of those agents with more outgoing communication channels and those connected to such agents, which is consistent with DeMarzo et al.'s (2003) theoretical predictions. Moreover, with experience, the estimated empirical weights of the different private signals correspond closely to those of the persuasion bias model. Hence, our data provide substantial support for the notion of persuasion bias. ${ }^{2}$

In a set of experiments conducted independently, Corazzini et al. (2012) find that the network structure plays a significant role in determining social influence, but that the most influential agents are not those with more outgoing links, as predicted by the persuasion bias hypothesis, but those with more incoming links. Their study presents data from a balanced and an unbalanced network that are different from those we study. They present an explanation of their results in the context of a generalization of the DeMarzo et al. (2003) model, suggesting a more general way in which bounded rationality might influence information updating in a network. In particular, the more general model takes into account

\footnotetext{
${ }^{2}$ Banerjee et al. (2013) and Mobius et al. (2015) contain some field evidence consistent with the DeGroot model. Our experiment also relates to a paper by Enke and Zimmermann (2013) on correlation neglect, which describes agents' tendency to overweight information, received from multiple sources, that is correlated due to its origin from the same source. They report a laboratory experiment in which subjects' exhibit correlation neglect and overweigh information that they receive repeatedly through multiple channels. They show experimentally that in such contexts many people neglect these correlations in the updating process and treat correlated information as independent.
} 
not only agents' outgoing links but also their incoming links - the idea being that those who "listen" to more people are potentially more influential. ${ }^{3}$

Given the qualitative discrepancy between the results of Corazzini et al. (2012) and our results, we conducted new experimental sessions using their unbalanced network. Our results are again consistent with the more restrictive notion of persuasion bias and do not need the additional flexibility of the more general model. In addition, we perform a simple counter-factual exercise by applying their model to our unbalanced network with the parameter estimates that are reported in Corazzini et al. (2013). For this comparison, we find that the persuasion bias model outperforms their model. Hence, across multiple networks, our study suggests greater concordance with the persuasion bias model of DeMarzo et al. (2003).

The remainder of this paper is structured as follows. In Section 2, we very briefly provide some theoretical background and describe the persuasion bias model of DeMarzo et al. (2003). Sections 3 and 4 present the design, hypotheses and results for Experiment 1, while Section 5 presents Experiment 2, which uses the network studied by Corazzini et al. (2012) to compare their model's predictions with those of DeMarzo et al. (2003). Section 6 concludes.

\section{Theoretical Background and Hypotheses for Experiment 1}

We now succinctly present the theoretical environment that we study experimentally, which is based on the model of DeMarzo et al. (2003). In all networks that we study, there is a set of four agents $N=\{1,2,3,4\}$ who all wish to find out the value of an unknown onedimensional parameter, $\theta$. There are sixteen periods of interaction between the four agents.

\footnotetext{
${ }^{3}$ Battiston and Stanca (2014) present additional evidence that the influence of an agent may be positively affected by the number of incoming links.
} 
The reason why we chose this particular number of periods is grounded in the persuasion bias model, which requires $\mathrm{N}^{2}$ periods for convergence (more detail provided below).

Before the first period of interaction, each agent obtains some initial information about $\theta$ in the form of a private noisy signal $\mathrm{x}_{\mathrm{i} 0}=\theta+\varepsilon_{\mathrm{i}}$, where $\varepsilon_{\mathrm{i}}$ is an error term with mean zero, independent across agents and normally distributed. Agents do not receive any other signals about the parameter. Each agent, i, assigns an initial precision to the information of each other agent, $\mathrm{j}$, represented by $\pi_{\mathrm{ijo}}$.

Starting with the initial information, agents communicate according to a communication network that can be represented as a directed graph indicating whether agent $\mathrm{i}$ listens to agent $\mathrm{j}$. We denote by $\mathrm{S}(\mathrm{i})$ the set of agents to which $\mathrm{i}$ listens, and by $\mathrm{q}_{\mathrm{ij}}$ the indicator function of $S(i)$, which takes the value of 0 if agent $i$ does not listen to agent $j$ and the value of 1 if he does; we consider that each agent listens to himself, e.g., $\mathrm{q}_{i i}=1$. We refer to the set $\mathrm{S}(\mathrm{i})$ as the listening set of agent $\mathrm{i}$ and to the function $\mathrm{S}$ as the listening structure.

DeMarzo et al. (2003) model communication and updating as follows. In the first communication period, agent i learns the signals of the agents in S(i). Given normality and agents' fixed assessment of the precision of others' information, a sufficient statistic for these signals is their weighted average, with weight given by the precisions. They denote this statistic by $\mathrm{x}_{\mathrm{i} 1}$ and refer to it as agent i's beliefs after one period of updating:

$$
\mathrm{x}_{\mathrm{i} 1}=\sum_{\mathrm{j}}\left(\mathrm{q}_{\mathrm{ij}} \pi_{\mathrm{ij} 0} / \pi_{\mathrm{ii} 0}\right) \mathrm{x}_{\mathrm{j} 0}
$$

where $\pi_{\mathrm{ii} 0}=\sum_{\mathrm{j}} \mathrm{q}_{\mathrm{ij}} \pi_{\mathrm{ij} 0}$ denotes the precision that agent $\mathrm{i}$ assigns to his own beliefs and $\mathrm{x}_{\mathrm{j} 0}$ represents j's initial beliefs. Equation (1) is called agent i's updating rule.

The updating rule (1) can be expressed in vector notation. Denote by $x_{t}$ the matrix whose $\mathrm{i}$-th row is the vector $\mathrm{x}_{\mathrm{it}}$ of agent $\mathrm{i}$ 's beliefs in communication period t. Denote also, by $\mathrm{T}$, the listening matrix with elements: 


$$
\mathrm{T}_{\mathrm{ij}}=\mathrm{q}_{\mathrm{ij}} \pi_{\mathrm{ijo}} / \pi_{\mathrm{ii1}}
$$

Then the updating rule can be expressed as $\mathrm{x}_{1}=\mathrm{T} \mathrm{x}_{0}$.

A very important simplifying assumption introduced at this point is that all precisions are the same. This converts each element of the listening matrix into something very simple: the ratio between the indicator $\mathrm{q}_{\mathrm{ij}}(0$ or 1$)$ and the total number of agents $\mathrm{j}$ that agent $\mathrm{i}$ listens to $(1,2,3$ or 4$)$. As a consequence of this simplification, the proposed updating consists simply of the averaging of others' estimates. ${ }^{4}$

We now briefly refer to the two key results of the DeMarzo (2003) model pertaining to the convergence process of the updating rule that we will use in our analysis below. First, their Theorem 1 states that if the set of agents is strongly connected and another technical assumption holds, then the vector of agents' social influences converges to the consensus given by the vector $\omega$ which is the unique solution $w=T w$, where $w$ is the "social influence" vector showing the weight of each agent's initial signal in the consensus. A set of agents being strongly connected simply means that every agent speaks to every other agent (directly or indirectly) and thus no agent is isolated from the others.

Second, their Theorem 2 states that, if agents follow the updating process introduced above, the beliefs to which agents converge are correct if and only if the network is balanced, i.e. if and only if, for all $\mathrm{i}, \sum_{\mathrm{j}} \mathrm{q}_{\mathrm{ji}} \mathrm{T}_{\mathrm{jj}}=1$. In words, if the sums of weights in the listening vectors are the same and equal to 1 for all agents. We now present the two networks studied in Experiment 1and directly apply the two theorems to these networks.

Figure 1 shows the "Balanced Network 1" used in our first experiment. The arrows indicate which agents send information to which other agents. For example, agent 1 sends

\footnotetext{
${ }^{4}$ In our experimental context. this assumption is a very reasonable one, since (as will become clearer below) our experimental subjects will not have much basis for attributing different precisions to distinct other agents.
} 
information to agents 2 and 4 and also receives information from agents 2 and 4 . This network is balanced, precisely because each agent talks and listens to the same number of other agents. Denoting by S(i) the set of agents to which agent i listens, we have, for the Balanced Network 1: $S(1)=\{1,2,4\} ; \quad S(2)=\{1,2,3\} ; \quad S(3)=\{2,3,4\} ; \quad S(4)=\{1,3,4\}$, including always the agent in question, i.e. each agent listens to himself.

From here we obtain the corresponding listening matrix $\mathrm{T}_{\mathrm{B} 1}=\begin{array}{cccc}1 / 3 & 1 / 3 & 0 & 1 / 3 \\ 1 / 3 & 1 / 3 & 1 / 3 & 0 \\ 0 & 1 / 3 & 1 / 3 & 1 / 3 \\ 1 / 3 & 0 & 1 / 3 & 1 / 3\end{array}$. To find the weights to which Theorem 1 above predicts behavior will converge, we solve the system $\mathrm{wT}=\mathrm{w}$ and find that the weight of the four signals in the consensus value to which the updating process converges is given by the vector, $\mathrm{w}_{\mathrm{B} 1}=(1 / 4,1 / 4,1 / 4,1 / 4)$, i.e. the social influence is the same for all four agents. In spite of the fact that the updating process does not take into account repetition, the process converges to an unbiased set of weights, because the network is balanced, consistent with Theorem 2 .

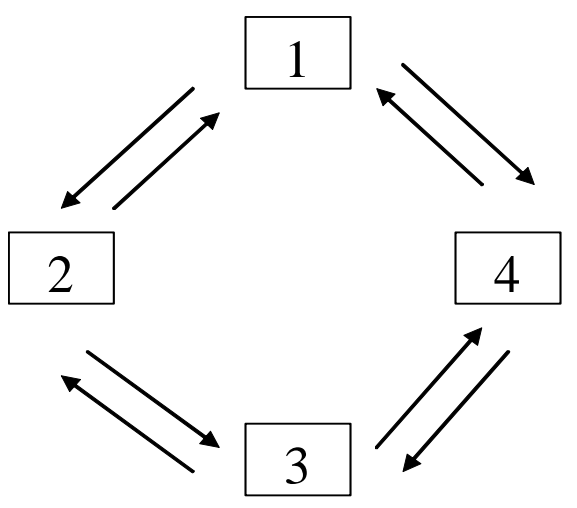

Figure 1: Balanced Network 1 
Figure 2 shows the "Unbalanced Network 1," where some agents speak and listen to a different number of other agents. In this case the sets of agents that agents listen to are the following: $S(1)=\{1,4\} ; S(2)=\{1,2,3\} ; S(3)=\{1,2,3,4\} ; S(4)=\{1,3,4\}$.

From here one can construct the corresponding listening matrix $\mathrm{T}_{\mathrm{UB} 1}=\begin{array}{cccc}1 / 2 & 0 & 0 & 1 / 2 \\ 1 / 3 & 1 / 3 & 1 / 3 & 0 \\ 1 / 4 & 1 / 4 & 1 / 4 & 1 / 4 \\ 1 / 3 & 0 & 1 / 3 & 1 / 3\end{array}$

. and obtain the following vector of social influence weights: $\mathrm{w}_{\mathrm{UB} 1}=(16 / 42,3 / 42,8 / 42$, 15/42) for agents 1, 2, 3 and 4 respectively. In percentage terms, this vector is approximately equal to $\mathrm{w}_{\mathrm{UB} 1}=(38 \%, 7 \%, 19 \%, 36 \%)$. Observe that persuasion bias does not propose that the influence weights directly reflect the number of agents an agent speaks to. Agents 3 and 4 both speak to two other agents, but the corresponding influence weights are $19 \%$ and $36 \%$ due to the fact that agent 4 speaks to agent 1 , who in turn talks to all three other agents.

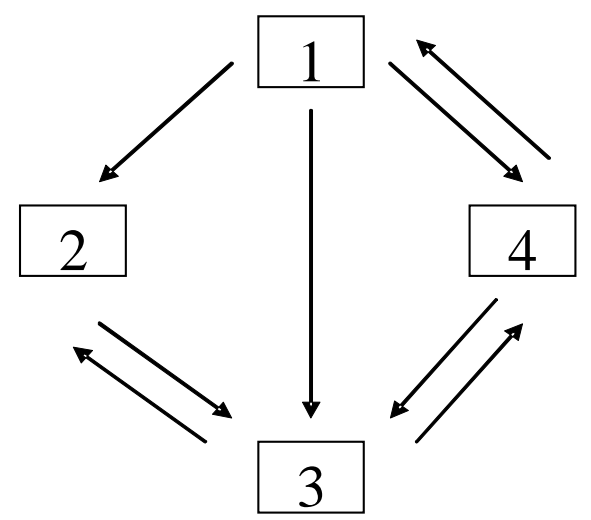

Figure 2: Unbalanced Network 1

We can then use the above predictions, from DeMarzo et al.'s (2003) model of persuasion bias, to generate precise hypotheses for an experimental test of information aggregation in the above two networks. Our first hypothesis is based on the prediction from 
DeMarzo et al. that, for both perfectly rational agents and to those affected by persuasion bias, the balanced network should produce no bias in consensus estimates.

\title{
Hypothesis 1: In Balanced Network 1, subjects will converge to a consensus estimate in which the four initial signals will carry equal weight.
}

Our second hypothesis uses the precise weights implied by DeMarzo et al.'s model for the specific unbalanced network in Figure 2.

\begin{abstract}
Hypothesis 2: In Unbalanced Network 1, subjects will converge to a consensus estimate in which the four initial signals will carry unequal weights. These weights will be as predicted by the persuasion bias model: 38\%, 7\%, 19\% and $36 \%$.
\end{abstract}

\section{Experiment 1: Procedures}

In Experiment 1, we study behavior in the two networks that we have just introduced, Balanced Network 1 and Unbalanced Network 1. The set-up was identical for all networks, with the exception of the links between subjects corresponding to the different communication networks. Each person participated only in one session with one particular network. The shape of the relevant network was common information. A graph of the network was visible to the participants at all times.

Each participant was a member of a fixed group of four subjects. ${ }^{5}$ Each group interacted consecutively in four blocks, each of which lasted for sixteen periods and was based on a new target value and private signals. This was to allow for learning of the task. At

\footnotetext{
${ }^{5}$ The instructions can be found in Appendix A.
} 
the beginning of a block, participants did not know the target value. Instead, each of the four subjects initially received a private signal drawn from a normal distribution centered on the target value. ${ }^{6}$ The signals were drawn separately for the different target values.

Once each participant had received his or her signal, then the sixteen periods of information exchange within the group for that block began. In each period, each agent in a group sent an estimate of the target parameter to all agents in his or her listening set. After receiving estimates from others according to the network structure, the group moved to the next period. All sixteen periods proceeded according to the same rules. The choice of sixteen periods was guided by the result in DeMarzo et al. (2003) that fully rational agents will converge to the true value in at most $\mathrm{N}^{2}$ periods of updating. Participants in the experiment should not necessarily be expected to behave rationally, meaning that their estimates may not fully converge; however, we needed to make a choice of horizon and we chose the minimal one that allows for convergence under the rational benchmark. ${ }^{7}$ Note that learning could not only take place within the sixteen periods of a block, but could also occur across the four blocks.

At the beginning of a block, subjects received their private signals through the computer interface. ${ }^{8}$ In each period, subjects could enter their estimate using the computer, and also observe the estimates entered by those in their listening set. Each subject was given a bundle of four record sheets, one for each block, and was instructed to write down his or her own estimate as well as the estimates of others in his or her listening set in each round. Each subject also received a sheet of paper depicting the communication network.

\footnotetext{
${ }^{6}$ Participants did not receive any additional signals in subsequent periods within a block.

${ }^{7}$ In related work, Corazzini et al. (2012) had four players and twelve periods and Battiston and Stanca (2014) four players and eight periods.

${ }^{8}$ The experiment was conducted using the software, z-tree (Fischbacher, 2007).
} 
In every period, each participant's payoff was determined by the distance between the estimate provided by the participant in that period and the actual target value in that block. Specifically, we implemented the payoff function, $\mathrm{P}=400-0.5 *$ (target number estimate $)^{2}$, where payoffs are denoted in Euro cents. In each block, one period was selected at random to count for payment. In principle, subjects' payoffs could be negative, meaning that they could lose part of the 5 Euro participation payment, though this was unlikely. We did not allow subjects to lose more than the participation payment.

Target values were randomly drawn from a uniform distribution over the range determined by taking the tenth highest number and the tenth lowest number from one hundred draws from a normal distribution with mean 0 and standard deviation 400 . This, in principle, allows for the target number to be any real number, though the likely ranges from which they are drawn are much more narrow and unlikely to yield very extreme numbers. Subjects were only told that the target number would be a real number and were not told anything else about how the number was determined. For robustness we investigated two complete parameter sets, i.e. two different sets of four target values with a corresponding set of signals for each of the four agents in the network. ${ }^{9}$ The two parameter sets were used in different sessions and can be found in Appendix B.

Experiment 1 was conducted at Pompeu Fabra University in Barcelona. Participants were undergraduate students at the university who were recruited with the on-line system ORSEE (Greiner, in press). Table 1 shows the distribution of groups per treatment for

\footnotetext{
${ }^{9}$ The ranges from which target values were drawn ended up being, in the Parameter Sets 1 and 2, respectively, $[-512.669,437.452]$ and $[-501.17,418.394]$.
} 
Experiment 1. Since each group is composed of 4 subjects, the total number of subjects in Experiment 1 is $144 .^{10}$

Table 1: Number of groups per treatment

\begin{tabular}{|c|c|}
\hline Treatment (Parameter Set \#) & Number of groups \\
\hline Balanced 1 (1) & 10 \\
\hline Balanced 1 (2) & 10 \\
\hline Unbalanced 1 (1) & 8 \\
\hline Unbalanced 1 (2) & 8 \\
\hline
\end{tabular}

\section{Experiment 1: Results}

We examine Hypotheses 1 and 2, pertaining to whether behavior converges toward an unbiased estimate of the true target value, in which the individual private signals receive equal weight, or whether they converge toward systematically biased consensus estimates that concord with the predictions of persuasion bias. Of course, while complete convergence is theoretical possible, we expect it to be unlikely, a priori, given the complexity of the task. For instance, in related experiments that use a different design, Battistin and Stanca (2014) do not observe complete convergence.

Nevertheless, we present three pieces of evidence that suggest estimates within groups converge across periods, even if complete convergence is rare. Figure 3 shows the evolution

\footnotetext{
${ }^{10}$ Two sessions, with four groups each, ended early because of computer problems. In one case, the experiment ended after period 51 (i.e., after 3 periods of Block 4); we use only Blocks 1 through 3 from this session. In the other case, the experiment ended after period 30 (i.e., after 14 periods of Block 2); since we have almost complete data for Block 2 in this session, we use both Blocks 1 and 2.
} 
over time of the average natural logarithm of the standard deviation of estimates within a group. We think that this is a sensible statistic to measure convergence, since it measures dispersion in individual estimates from the group mean estimate, using the natural logarithm to reduce the influence of outliers. The different panels show different aggregations of the data. In all cases, there is a downward trend, indicating that the estimates move closer together, on average, across periods within a block. Panel A shows this is true in the aggregated data. Panel B separates the data by block, revealing slightly weaker convergence in Block 3, but also a downward trend in all four blocks and little systematic evidence of learning across blocks. Panel $\mathrm{C}$ shows similarly decreasing trajectories across periods when dividing the data by parameter set. Finally, Panel D compares behavior for the two different networks, suggesting convergence is slightly stronger in the case of the unbalanced network. However, in all cases, there is a clear downward trend.

An additional piece of evidence indicating a convergence process is the results of panel regressions, with group-level random effects, of the natural logarithm of the standard deviation of group estimates on the period within a block. Using all data jointly, we find a negative coefficient, -0.177 with a standard error of 0.014 , which is statistically significant at the $1 \%$ level. Moreover, separate regressions for the balanced and unbalanced networks, the two parameter sets and for each of the four blocks show very similar results. In all cases, there is a significant downward trajectory across periods within a block.

Finally, Figure 4 presents a different perspective of the convergence process that facilitates identifying possible heterogeneity across groups. It shows the evolution over time of the proportion of groups with standard deviations of estimates below five different thresholds. One can see that, for any threshold, there is an upward tendency over time. For example, in the first period, few groups have standard deviations below 20; this is not surprising, as the standard deviations of private signals are greater than 20 in almost all cases 
(see Appendix B). However, by the end of the block, almost all groups have standard deviations below that threshold. Similarly, virtually no groups start off with standard deviations below 10, but roughly three-quarters of groups are below this threshold by the end of the block. While the proportion of groups with standard deviations below 1 is never high, this portion also increases across periods within a block.

To summarize, our data are not fully consistent with the hypothesis that behavior converges to a consensus. In fact, there are zero instances in which all four estimates are identical by the end of a block. However, relaxing this strict criterion, we see considerable evidence that estimates move in the direction of consensus. For example, the proportion of groups with standard deviations below 0.1 increases from zero in the first period of a block to 6.3 percent by the sixteenth period. More generally, we see widespread movement toward similar estimates across periods in almost every way we look at the data.

We next turn to an analysis of what the average estimate in groups converge toward and whether this central tendency is consistent with Hypotheses 1 and $2 .{ }^{11}$ For this, we use the final 8 periods in a block, where Figures 3 and 4 show most of the convergence has taken place. We then calculate, as a measure of the central tendency in a group's estimates, the average estimate in each group for all players across all 8 periods. We then estimate average "bias" in group estimates, by comparing the average estimate in the final 8 periods to the mean of the private signals received by the four group members; specifically, our measure of bias is the average estimate in the group minus the average of the signals. That is, a group that converges to mean estimates equal to the average of the four private signals shows zero

\footnotetext{
${ }^{11}$ Evidence of convergence toward better informed estimates can be seen by comparing the earnings from the first half of a block (periods 1-8) with the second half (periods 9-16). Average payoffs rose from -1.82 Euro to 2.31 Euro, while median payoffs rose from 3.59 Euro to 3.76 Euro. Comparing the first and last period, we see that the average (median) payoff also increased, from -13.32 (2.27) Euro to 2.56 (3.74) Euro. The discrepancies between mean and median earnings are due to the quadratic loss term in the payoff function and the large impact of occasional extreme estimates on the mean. Recall that subjects' losses were bounded, such that subjects could not leave the experiment with a negative balance.
} 
bias, while a group that converges to a mean estimate that is 4 greater than the rational benchmark shows a bias of 4 .

Figure 5 shows the median bias across groups, in the average estimates for the final eight periods of each block. Specifically, for each group, we look at the mean of the 32 estimates in the final eight periods of a block. We identify the bias as the difference between the actual mean group estimate and the rational benchmark of the average of all the private signals. For the balanced network the predicted values of bias are zero, according to Hypothesis 1. For the unbalanced network, persuasion bias predicts a level of bias represented by the differential weights on the private signals; this predicted bias is provided by the solid line. The dashed lines show the observed data, by the type of network. Panel A provides this information for Parameter Set 1, while Panel B does so for Parameter Set 2. Both panels suggest that the estimates toward which participants tend are generally directionally in line with the predictions of persuasion bias. There is almost always greater bias in the behavior in the unbalanced networks and this generally concords-in six of eight cases - with the directional prediction of the persuasion bias model. In fact, the concordance with the persuasion bias predictions in the first three blocks for Parameter Set 1 and the first two blocks for Parameter Set 2 is quite striking.

To put the analysis of the bias on a more solid basis we use regression analysis. Table 2 shows, in columns 1 and 2, the results of regressions with subject fixed-effects, for the Balanced Network 1 and the Unbalanced Network 1, respectively. Individual subjects' estimates are regressed on the value of the four private signals received by group members in that block. In column 1, the results for the balanced network show that the coefficients for all four signals are significant and appear to be close to the predicted and unbiased weights of 0.25. The results of an F-test show that the null hypothesis that all four coefficients are equal to 0.25 cannot be rejected ( $\mathrm{p}=0.197$, two-sided). We can also test whether the estimated 
weights for the Balanced Network 1 are similar to those predicted for the Unbalanced Network 1 by the persuasion bias model. Under the model, this should not be the case. An Ftest rejects, at marginally significant levels, the null hypothesis that the estimated weights for the Balanced Network 1 are equal to those predicted for the Unbalanced Network 1 ( $\mathrm{p}=$ $0.056)$.

Table 2: Regression results

\begin{tabular}{|c|c|c|c|}
\hline $\begin{array}{l}\text { Independent Variables } \\
\text { and Other Information }\end{array}$ & $\begin{array}{l}\text { Balanced Network } 1 \\
\text { Individual Estimate } \\
\text { (1) }\end{array}$ & $\begin{array}{l}\text { Unbalanced Network } 1 \\
\text { Individual Estimate } \\
\text { (2) }\end{array}$ & $\begin{array}{c}\text { Unbalanced Network } 2 \\
\text { Individual Estimate } \\
\text { (3) }\end{array}$ \\
\hline Signal 1 & $\begin{array}{c}0.294 * * * \\
(0.052)\end{array}$ & $\begin{array}{c}0.355^{* * *} * \\
(0.060)\end{array}$ & $\begin{array}{c}0.464 * * * \\
(0.055)\end{array}$ \\
\hline Signal 2 & $\begin{array}{c}0.225 * * * \\
(0.057)\end{array}$ & $\begin{array}{c}0.065 \\
(0.070)\end{array}$ & $\begin{array}{c}0.027 \\
(0.076)\end{array}$ \\
\hline Signal 3 & $\begin{array}{c}0.194 * * * \\
(0.032)\end{array}$ & $\begin{array}{c}0.219 * * * \\
(0.046)\end{array}$ & $\begin{array}{c}0.213 * * * \\
(0.045)\end{array}$ \\
\hline Signal 4 & $\begin{array}{c}0.279 * * * \\
(0.051)\end{array}$ & $\begin{array}{c}0.343 * * * \\
(0.056)\end{array}$ & $\begin{array}{c}0.289 * * * \\
((0.057)\end{array}$ \\
\hline Constant & $\begin{array}{l}1.340 \\
(1348)\end{array}$ & $\begin{array}{l}-1.673 \\
(1.695)\end{array}$ & $\begin{array}{l}-0.487 \\
(2.212)\end{array}$ \\
\hline $\begin{array}{c}\text { Number of } \\
\text { observations }\end{array}$ & 2432 & 1760 & 2432 \\
\hline R-squared & 0.987 & 0.992 & 0.9835 \\
\hline $\begin{array}{l}\text { Number of Unique } \\
\text { Subject IDs }\end{array}$ & 80 & 64 & 76 \\
\hline
\end{tabular}

The regressions above use as dependent variables the estimate provided by a subject in a period, using the final 8 periods (9-16) in a block. We include subject fixed-effects and cluster standard errors by groups.

For the unbalanced network, the regression results in column 2 show that ordering of the estimated weights is the same as that of the weights predicted by the persuasion bias model. Specifically, we compare the estimated weights for the Balanced Network 1 with the prediction of DeMarzo et al. (2003) for the Unbalanced Network 1, which attributes 38\%, $7 \%, 19 \%$ and $36 \%$ of the weights to signals $1,2,3$ and 4 , respectively. We find that an F-test fails to reject the null hypotheses that the estimated weights for the Unbalanced Network 1 are equivalent to the above weights predicted by persuasion bias ( $\mathrm{p}=0.136$, two-sided). In 
contrast, an F-test rejects the null hypothesis of equal weights for the Unbalanced Network 1 $(\mathrm{p}=0.038$, two-sided $)$.

Hence, taken together, our four tests document that the estimated weights for each of the two networks are consistent with those predicted by the DeMarzo et al. (2013) model for that network and inconsistent with those predicted for the other network.

What can we conclude with respect to our Hypotheses 1 and 2? First, for both networks, we do not see full convergence to a consensus estimate. However, we do see clear evidence of movement over time toward estimates that are more similar within groups. Hence, despite the lack of complete convergence to a common estimate, there is clearly partial convergence toward more similar estimates. More importantly, this movement tends to be in the direction of the weights on initial private information as predicted by DeMarzo et al.'s model of persuasion bias.

\section{A More General Model and Experiment 2}

After having conducted Experiment 1, we became aware of a related paper by Corazzini et al. (2012). This paper presents the results of experiments on persuasion bias using a balanced network and an unbalanced one, both different from the ones we use in Experiment 1. They find results that are not in line with persuasion bias. Instead, in their data, "social influence depends not only on being listened to by many others, but also on listening to many others" (p. 1276). In addition, they present a model of a generalized boundedly rational updating rule and show that persuasion bias and the behavior observed in their experiment can both be seen as the outcomes of special cases of that updating rule.

The interpretation provided by Corazzini et al. (2012) is indeed an intriguing one. An important question is what the precise behavioral mechanism is that underlies the rule that they use to explain the behavior in their experiment. Specifically, the observation of the 
network structure may lead some individuals to give more weight to the input of somebody who listens to many players, guided by the notion that those players have a lot of information. In this sense, the idea that influential listeners' information should carry more weight can be interpreted as players being somewhat more rational than under DeMarzo et al.'s persuasion bias model. That is, rather than simply treating all information as equally informative, such individuals look at the network structure to infer who has better information. Of course, an important question is why such behavior does not arise in our Experiment 1 .

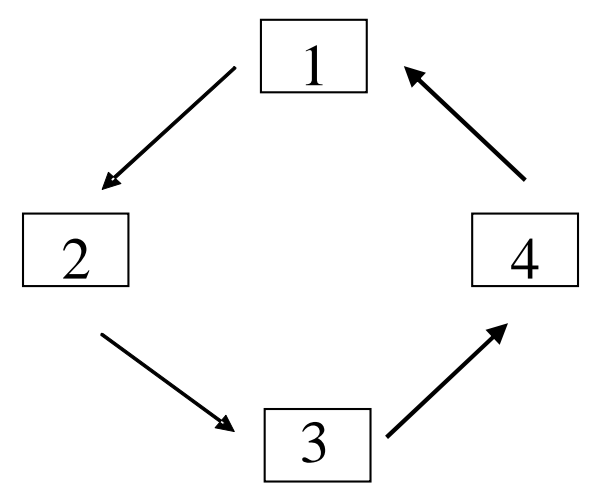

Figure 3: Balanced Network 2

One possible source of the difference between their results and ours is that the two experiments employ different networks. Figure 3 shows "Balanced Network 2," which is the balanced network studied by Corazzini et al. (2012). The listening sets for this network are $S(1)=\{1,4\} ; S(2)=\{1,2\} ; S(3)=\{2,3\} ; S(4)=\{3,4\}$. The listening matrix and the vector of influence weights are given by $\mathrm{T}_{\mathrm{B} 2}=\begin{array}{cccc}1 / 2 & 0 & 0 & 1 / 2 \\ 1 / 2 & 1 / 2 & 0 & 0 \\ 0 & 1 / 2 & 1 / 2 & 0 \\ 0 & 0 & 1 / 2 & 1 / 2\end{array}$ and $\mathrm{w}_{\mathrm{B} 2}=(1 / 4,1 / 4,1 / 4,1 / 4)$, respectively. The regression results reported in Corazzini et al. (2012) for Balanced Network 2 find that the values of the estimated coefficients vary between 0.15 and 0.34 , but that the null hypothesis of equality of equal weights for the four coefficients cannot be rejected. This 
finding supports our Hypothesis 1 above, and provides further evidence that behavior in balanced networks is consistent both with the persuasion bias model and with rational information aggregation.

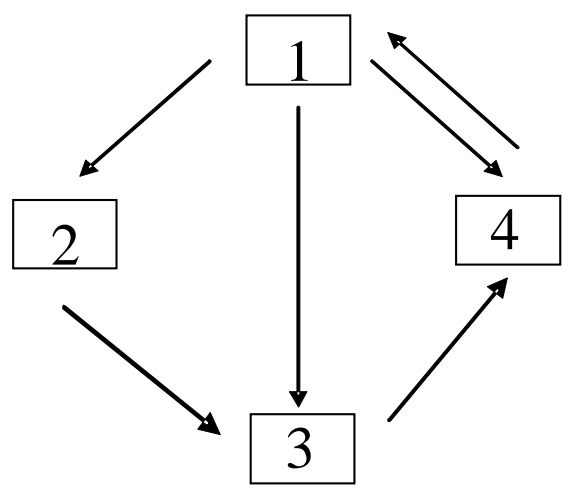

Figure 4: Unbalanced Network 2

Figure 4 shows "Unbalanced Network 2," which is the unbalanced network studied in Corazzini et al. (2012). Here, the listening structure is given by: $S(1)=\{1,4\} ; S(2)=\{1,2\}$; $S(3)=\{1,2,3\} ; \quad S(4)=\{1,3,4\}$ and the listening matrix is $\mathrm{T}_{\mathrm{UB} 2}=\begin{array}{cccc}1 / 2 & 0 & 0 & 1 / 2 \\ 1 / 2 & 1 / 2 & 0 & 0 \\ 1 / 3 & 1 / 3 & 1 / 3 & 0 \\ 1 / 3 & 0 & 1 / 3 & 1 / 3\end{array}$. The corresponding influence vector, according to DeMarzo et al.'s persuasion bias model is $\mathrm{w}_{\mathrm{UB} 2}$ $=(42 \%, 10 \%, 16 \%, 32 \%)$. Agent 1 is the most influential one, since he is the only one that speaks to all three others. Agent 2 is the least influential one, since he only speaks to agent 3 , who only indirectly reaches agent 1 . Agent 4 is the second most influential one; like agents 2 and 3 he only speaks to one other agent, but this agent turns out to be agent 1 who speaks to all three others.

The weights that Corazzini et al. (2012) find for signals 1 to 4 in their data analysis are, respectively, 27\%, 20\%, 22\% and 31\% (see Table 1 in Corazzini et al. (2012)). An F-test rejects the null hypothesis of equality of the weights. This configuration is certainly quite 
distinct from the one predicted by the persuasion bias model of DeMarzo et al. (2003), of $42 \%, 10 \%, 16 \%$ and $32 \%$. This discrepancy is intriguing and led Corazzini et al. (2012) to formulate the more general model described above.

To provide insights into possible sources of the discrepancy between Corazzini et al.'s (2012) findings and ours, we employ two approaches. First, we derive the predictions of their model for our Unbalanced Network 1, from our Experiment 1, to see how well their model does in explaining our independent data. Second, we conduct new experimental sessions using the unbalanced network studied by Corazzini et al. (2012), Unbalanced Network 2, to see if their result replicates with our procedures.

To compare the predictions of their model to those of ours for Experiment 1, we use two different weight vectors that correspond to two different parameterizations-specifically, two different values of $\rho$-of the model proposed by Corazzini et al. (2012). In their model, this parameter measures the degree to which players overweigh the information of those who receive more information from others in the network. A value of $\rho$ equal to zero implies the persuasion bias model of DeMarzo et al. (2003), while a value of $\rho$ equal to one implies that players weigh signals they receive by the number of incoming network connections of the person sending that signal. For $\rho=0.82$, the value that maximizes explanatory power for the weights they observe in their experiment, the vector of weights for the Unbalanced Network 1 from our Experiment 1 is $21 \%, 13 \%, 33 \%$ and $33 \%$. For $\rho=1$, the weights are $25 \%, 10 \%$, $30 \%$ and $34 \%$. Observe that both vectors are quite distinct from the estimated weights we observe in the data, of $36 \%, 7 \%, 22 \%$ and $34 \%$ (see Table 1, column 2), which appear to be more similar to those predicted by DeMarzo et al.'s persuasion bias model $(38 \%, 7 \%, 19 \%$, $36 \%)$.

To evaluate the closeness of the vector of weights that we estimate from our data with that of the persuasion bias model of DeMarzo et al. (2003) and the influential listeners model 
of Corazzini et al. (2012), we use distance functions. The left-hand side of Table 3 shows the Euclidean and Taxicab distances of our estimates to the two models for the Unbalanced Network 1 studied in Experiment $1 .^{12}$ One can see that our estimated weight vector is much closer to the prediction of DeMarzo et al.'s (2003) persuasion bias model than to the Corazzini et al. (2012) model for the two specific parameter values we consider.

Table 3: Distances of the estimated weight vector from the models

\begin{tabular}{|c|c|c|c|c|c|}
\hline \multicolumn{5}{|c|}{ Euclidean Distances } \\
\hline DeMarzo & $\begin{array}{c}\text { Corazzini } \\
(\rho=1)\end{array}$ & $\begin{array}{c}\text { Corazzini } \\
(\rho=0.82)\end{array}$ & DeMarzo & $\begin{array}{c}\text { Corazzini } \\
(\rho=1)\end{array}$ & $\begin{array}{c}\text { Corazzini } \\
(\rho=0.82)\end{array}$ \\
\hline 0.0017 & 0.0188 & 0.0374 & 0.0117 & 0.0437 & 0.0681 \\
\hline \multicolumn{7}{|c|}{ Taxicab Distances } \\
\hline Unbalanced Network 1 & Unbalanced Network 2 \\
\hline DeMarzo & $\begin{array}{c}\text { Corazzini } \\
(\rho=1)\end{array}$ & $\begin{array}{c}\text { Corazzini } \\
(\rho=0.82)\end{array}$ & DeMarzo & $\begin{array}{c}\text { Corazzini } \\
(\rho=1)\end{array}$ & $\begin{array}{c}\text { Corazzini } \\
(\rho=0.82)\end{array}$ \\
\hline 0.0751 & 0.2265 & 0.3329 & 0.2031 & 0.3544 & 0.3950 \\
\hline
\end{tabular}

We now move to our second comparison between the two models-Experiment 2, in which we study Unbalanced Network 2, using the same procedures and parameter sets as in our Experiment $1 .{ }^{13}$ We had 10 groups of participants with Parameter Set 1 and 9 groups with Parameter Set 2, a total of 76 participants. Experiment 2 was conducted in the LINEEX lab at

\footnotetext{
12 The Taxicab distance between two points is the sum of the absolute differences of their Cartesian Coordinates.

${ }^{13}$ We did not use Balanced Network 2 in this study, since both our results and those of Corazzini et al. (2012) are consistent with all four signals receiving equal weights in balanced networks.
} 
the University of Valencia. Participants were undergraduate students at the university and recruiting was again done using the electronic system ORSEE (Greiner, in press).

Figures 6 and 7 are analogous to Figures 3 and 4 for Experiment 1. Figure 6 shows the evolution over time of the average natural logarithm of the standard deviation of guesses within a group for the Unbalanced Network 2, and Figure 7 shows the evolution over time of the proportion of groups with standard deviations of estimates below five different thresholds for the Unbalanced Network 2. The main features to note in both figures at this point is that the patterns are rather similar for Experiments 1 and 2. Regression analysis confirms the impression one gets from inspecting Figures 6 and 7. The results of random-effects panel regressions of the natural logarithm of the standard deviation of group estimates on the period within a block, using all data from Experiment 2, jointly finds a negative coefficient, -0.086, with a standard error of 0.015 , significant at the $1 \%$ level. Moreover, as with Experiment 1 , we similarly find significant negative time trends in all separate analyses by parameter sets and by blocks. Hence, as was the case in Experiment 1, our data indicate some degree of convergence toward similar estimates within a group, even if such convergence is imperfect.

We next use regression analysis to estimate the vectors of weights on the private signals that reflect the central tendency in group estimates. The third column in Table 2 shows regressions results for our Experiment 2 comparable to those for the two networks in Experiment 1 . The point estimates appear to be quite distinct from each other and indeed an F-test strongly rejects the null hypothesis of equality of coefficients at the $1 \%$ level. Our estimated values for the vector of weights are $46 \%, 3 \%, 21 \%$ and $29 \%$. The ordering of the magnitudes of these weights is in line with the persuasion bias model, which predicts weights of $42 \%, 10 \%, 16 \%$ and $32 \%$. The right side of Table 3 shows that, like for our Experiment 1 , the estimated weight vectors are closer to the predictions of DeMarzo et al.'s (2003) 
persuasion bias model — using either Euclidian or Taxicab distances—than to the predictions of either version of the Corazzini et al. (2012) model.

As we did for Experiment 1, we can also test for the differences between the regression results and the predicted weights of the different models. We start with the test for equality of the regression coefficients with the weights predicted by the DeMarzo et al. (2003) model. An F-test finds that we cannot reject the hypothesis of equality ( $p=0.286$, twosided). We can also test for the equality of the estimated coefficients with the two variants of the Corazzini et al. (2012) model. Two-sided F-tests reject both variants of the model at the 5\% level $(\rho=1: p=0.024, \rho=0.82: p=0.018)$. Hence, Experiment 2, which studies behavior in the Unbalanced Network 2 first studied by Corazzini et al. (2012) using our procedures, lends further support for the predictions of the persuasion bias model, in contrast with the two versions of the "influential listeners" model that best accounts for their data.

\section{Concluding Remarks}

We provide several experimental results that are consistent with the predictions of a model in which communication through networks yields biased information aggregation. Our findings provide support for the prediction that people with more outgoing links and those connected to such people exert greater influence on group beliefs. Moreover, our results also yield estimated influence weights that are very close to those predicted by the model of DeMarzo et al. (2003).

The fact that the results from our Balanced Network 1 are consistent with these predictions is perhaps not surprising. However, the results from two different unbalanced networks are also consistent with persuasion bias. In addition we confront the data with a more general model proposed by Corazzini et al. (2012) and do not find it to explain the data better than persuasion bias. One of the possible explanations for this disparity is that the 
specifics of our experimental procedures are more closely related to the set-up of the theoretical framework of DeMarzo et al. (2003). ${ }^{14}$ As a consequence, the task that participants face may be cognitively more demanding than the one in Corazzini et al. (2012). In addition, the salience of the monetary incentive is lower with our procedures due to the variability of the signals; it is possible for participants to earn zero even when optimally processing the information they receive, since the averages of the signals are generally different from the target value. Furthermore, in our experiment participants did not know the variance of the distribution. All these features of our design may have led participants to make little cognitive effort and instead rely on the very simple updating rule that leads to persuasion bias, without attempting to exploit the information about the network structure in some way.

On the basis of our findings, we conclude that the persuasion bias model represents a good benchmark for the study of information aggregation. The model is based on a simple, intuitively defensible bias in information processing and we demonstrate that it generates predictions that are supported empirically in a careful test. In our data, the basic DeMarzo et al. (2003) model does rather well and the additional flexibility of the Corazzini et al. (2012) is not needed. To us this conclusion does not come as a surprise, since from the start the persuasion bias model seemed to us as a simple, perhaps very mechanical, but nevertheless natural way to behave. Of course, future research may produce results that may be at odds with persuasion bias, requiring the additional flexibility of the Corazzini et al. (2012) model

\footnotetext{
${ }^{14}$ We thank a reviewer for suggesting this explanation. Relating the implication of using different procedures to the issue of external validity, perhaps one can say that natural environments will tend to be quite complex with many distractions that yield different cognitive approaches to theoretically similar problems. This may account for why one might observe particular biases in some situations but not others. At the same time, of course, agents in natural settings may often have higher incentives to behave rationally.
} 
or an altogether new model. But, in at least one test, the assumptions and predictions of persuasion bias appear vindicated. 
Acknowledgements: Financial support by the Spanish Ministerio de Ciencia e Innovación(Grant: ECO2011-29847-C02-01), the Generalitat de Catalunya (Grant: 2009 SGR 820), the Barcelona GSE Research Support Program and Consolider-Ingenio is gratefully acknowledged. Aniol Llorente-Saguer and Julian Rode provided excellent research assistance.

\section{REFERENCES}

Acemoglu, D., Dahle, M., Lobel, I., Ozdaglar, A., 2011. Bayesian learning in social networks. Review of Economic Studies 78(4), 1201-1236.

Banerjee, A., Chandrasekhar, A., Duflo, E., Jackson, M., 2013. The diffusion of microfinance. Science 341(1644).

Battiston, P., Stanca, L., 2014. Boundedly rational opinion dynamics in directed social networks: theory and experimental evidence. Working Paper No. 267. Department of Economics, Management and Statistics, University of Milan - Biccoca.

Corazzini, L., Pavesi, F., Petrovich, B., Stanca, L., 2012. Influential listeners: An experiment on persuasion bias in social networks. European Economic Review 56, 1276-1288.

DeGroot, M., 1974. Reaching a consensus. Journal of the American Statistical Association $69,118-121$.

DeMarzo, P., Vayanos, D., Zwiebel, J., 2003. Persuasion bias, social influence and unidimensional opinions. Quarterly Journal of Economics, 909-968.

Enke, B., Zimmermann, F., 2013. Correlation neglect in belief formation. CESIfo Working Paper 4483.

Falk, A., Heckman, J., 2009. Lab experiments are a major source of knowledge in the social sciences. Science, 326(5952), 535-538.

Fischbacher, U, 2007. z-Tree: Zurich toolbox for ready-made economic experiments. Experimental Economics, Springer, vol. 10(2), pages 171-178, June.

Gale, D., Kariv, S. 2003. Bayesian learning in social networks. Games and Economic Behavior 45 (2), 329-346. 
Golub, B., Jackson, M.O., 2010. Naïve learning in social networks: convergence, influence, and the wisdom of crowds. American Economic Journal: Microeconomics 2 (1), 112149.

Greiner, B. In press. Subject pool recruitment procedures: organizing experiments with ORSEE. Journal of the Economic Science Association.

Mobius, M., Phan, T., Szeidl, A., 2015. Treasure hunt: social lerning in the field. Working Paper. 


\section{APPENDIX A: Instructions}

\section{General Information}

This is an experiment in decision-making. During the experiment, you will accumulate money. In addition to a participation fee of $5 €$, you will receive the amount you accumulate in cash after the experiment is finalized. The exact amount you receive will be determined during the experiment and will depend on your decisions and/or the decisions of other participants.

If you have any questions during the experiment, please raise your hand and wait for an experimenter to come to you.

Please do not try to communicate with other participants during the experiment.

At the bottom of this screen, you will see a letter (A, B, C, or D) and a number $(1,2$, 3 , or 4). This combination of letter and number is your participant number for the experiment. Your participant number will be the same for the entire experiment. This number is private and should not be shared with anyone. Please record this number at your stack of record sheets.

Your participation number ......

\section{Periods, Rounds, Groups, and Roles:}

During this experiment, you will be in a group with three other participants. You will be grouped with the three other people with the same letter in their participant number. That is, everyone with a letter " $A$ " in his/her participation number will be in the same group, everyone with the letter "B" will be in the same group, and so on. Throughout the experiment, you will be grouped with the same three participants.

In the experiment, you will participate in 4 rounds of an activity. Each round will consist of 16 periods. That is, during the experiment you will participate in Rounds 1 through 4, and each of these rounds will consist of Periods 1 through 16.

For each round, we will randomly select one of the 16 periods for which to pay you. Since you will not know which period will be selected for payment until the end of the experiment, every period could influence your earnings for the experiment.

\section{Round Instructions:}

In each round, a number will be drawn at random for your group. This number will be a real number and can include three decimal places. The range from which we draw the number may change from round to round. You will not be given any other information about how the number is determined. This number will be referred to as the "target number" for your group for that round. 
At the beginning of each round, every member of your group will receive a "private estimate" of the target number. This private estimate will consist of the target number, plus or minus a random number. For each member of your group, the random number will be drawn from the normal distribution, with an average of zero. This means that your estimate will be related to the target number, but only imperfectly so. That is, your estimate will give you an idea of the target number, but will most likely not be the same as the target number. To be more precise, the private estimates will be normally distributed around the target number.

Each of you will receive a different private estimate. Each of the estimates will be drawn independently from the normal distribution around the target number. That is, for each of you we will start with the target number and then add a random number drawn from the normal distribution. Your private estimates will therefore be related to each other and to the target number, but only imperfectly so.

Are there any questions about the target number and estimates?

\section{Period Instructions:}

In each period, you will enter your guess of the target number. You will do so by entering a number (of up to 3 decimal places). The computer will prompt you for your guess and will then ask you to confirm this guess. If you want to enter a guess with a negative sign, make sure that you do not forget to put the negative sign.

In each period, your payoff will be determined by how close your guess is to the target number. That is, we will compare your guess with the target number. The closer your guess is to the target number, the higher your payoff will be for that period. Note that you will not be able to see either the target value or your payoff for each period until the end of the round.

\section{IMPORTANT: YOU NEED TO USE THE DECIMAL POINT (.) TO ENTER THE DECIMALS.}

\section{Information Instructions:}

At the end of each period, you will receive information on the guesses of other participants. Each of you will be able to observe the guess entered in that period by at least one other participant. Some of you may receive more information than others.

There are four participants in each group, with numbers from 1 to 4 . The information received by each participant is presented in the table below. For each participant (row), the columns with an " $x$ " indicate which guesses that participant is able to observe. Note that each participant is able to observe his or her own guess. In addition, every participant will be able to observe the guess of at least one other participant. For example, in addition to his/her own guess, Participant 3 can observe the guesses of the rest of the participants whereas Participant 1 can observe only the guess of Participant 4. 


\begin{tabular}{|c|c|c|c|c|}
\hline & \multicolumn{3}{|l|}{ Observes the guess of participant: } \\
\hline & Participant 1 & Participant 2 & Participant 3 & Participant 4 \\
\hline Participant 1 & X & & & X \\
\hline Participant 2 & X & X & X & \\
\hline Participant 3 & X & X & X & X \\
\hline Participant 4 & X & & X & X \\
\hline
\end{tabular}

Therefore, the only information you will have on the target number is your private estimate as well as the guesses you receive from the other participants.

To summarize, in each period you will make a guess, this guess will determine your payoff for that period, and you will then observe the guesses of some of the other members of your group. Once this takes place, you will continue to the next period.

After 16 periods, the round will end, and you will be able to see the target number for that round, as well as your payoff in each of the 16 periods. At the end of the experiment, one of these 16 periods will be selected at random to determine your earnings for that round. You will be paid your earning across all 4 rounds, in addition to the $5 €$ participation fee, in cash.

Are there any questions?

\section{Payoff Instructions:}

Your payoff in every period will be determined by the following formula (in cents):

$400-0.5 *(\text { target number }- \text { guess })^{2}$.

To make sure everyone understands the payoff formula, let us go through a few examples:

- Example 1: If your guess is exactly the same as the target number, then your payoff in that period will be: $400-0.5 *(0)=400$ cents $(4 €)$.

- Example 2: If the target number is 100.0 and your guess is 80.0 , then your payoff will be:

$$
400-0.5 *(100-80)^{2}=400-0.5 *(400)=400-200=200 \text { cents }(2 €) .
$$

- Example 3: If the target number is -60.0 and your guess is -20.0 , then your payoff will be:

$400-0.5 *(-60.0-(-20.0))^{2}=400-0.5 *(20.0-60.0)^{2}=400-0.5 *(1600)=400-$ $800=-400$ cents $(-4 €)$

Please note three things about how your payoff will be determined. 
First, a simple way to think about your payoff is that you start off with 400 cents, but lose money for the distance between your guess and the target number. Therefore, you want your guess to be as close as possible to the target number.

Second, remember that you will only receive payment for one of the 16 periods in a round. Since you will not know which period this will be until the end of the experiment, every period is equally likely to count towards determining your earnings.

Third, notice that it is possible to lose money in a period if your guess is far from the target number. If your payoff in a period is negative, and if that period is selected to count for that round, then the amount you lose will be subtracted from the amount that you accumulate in other rounds. This means that you may lose some of your participation fee if the sum of your payoffs for all 4 rounds is negative. However, we have designed the experiment so that this is very unlikely to be the case if you make decisions carefully. If you notice that you are accumulating negative payoffs regularly, please raise your hand so that we can make sure that you understand how payoffs are determined. Also, please double-check your guess before confirming it, since entering it incorrectly may cause you to lose a significant amount of money.

Are there any questions about your payoffs?

\section{Quiz (Part 1)}

Before proceeding to the experiment, we would like to ask you to answer a few questions to make sure that everyone understands the instructions. Please answer each of the following questions. Once you have completed the quiz, please wait for the experimenter to ask you to proceed.

1. The experiment consists of 4 rounds. Each round consists of 16 periods.

$$
\text { True False }
$$

2. At the beginning of each round, everyone in your group will be shown the target number.

True False

3. The target number in a round will be the same for everyone in your group.

$$
\text { True False }
$$

4. The private estimate you receive will be the same for everyone in your group.

$$
\text { True False }
$$

5. In each period, your payoff will be based on how close your guess is to the target number.

True False

6. At the end of every period, each of you will observe the guess of at least one other person in your group.
True
False

Quiz (Part 2) 
For the next three questions, remember that the formula for your payoff in a period is:

$400-0.5 *$ (target number - guess $)^{2}$.

7. Suppose that in a period your guess is 50.0. If the target number is 50.0, then your payoff for that period is:

8. Suppose that in a period your guess is 200.00 . If the target number is 190.0 , then your payoff for that period is: .....

9. Suppose that in a period your guess is 500.00 . If the target number is 470.0 , then your payoff for that period is:

\section{Final Instructions:}

We are now ready to begin the experiment. We will proceed through 4 rounds, each of which will consist of 16 periods. At the end of each round, you will find out the target number as well as your payoff for each of the 16 periods. At the end of the experiment, one of these periods will be selected at random for each round to determine your earnings.

At the beginning of each round, please record your private estimate on your record sheet. Then, in each period, please also record your guess and the information you receive regarding the guesses of others.

If you have a question from this point on, please raise your hand and wait for the experimenter. 
APPENDIX B: Target Values and Private Signals

\section{Information set 1:}

\begin{tabular}{|c|c|c|c|c|}
\hline Target Value & \multicolumn{4}{|c|}{ Private Signals } \\
\hline & Agent 1 & Agent 2 & Agent 3 & Agent 4 \\
\hline 81.405 & 63.884 & 85.355 & 93.682 & 78.521 \\
\hline-21.6 & -3.518 & -27.486 & -51.65 & -12.146 \\
\hline 124.16 & 119.512 & 95.831 & 146.349 & 153.696 \\
\hline 6.327 & 35.719 & 2.553 & 25.543 & -11.991 \\
\hline
\end{tabular}

\section{Information Set 2:}

\begin{tabular}{|c|c|c|c|c|}
\hline Target Value & \multicolumn{4}{|c|}{ Private Signals } \\
\hline & Agent 1 & Agent 2 & Agent 3 & Agent 4 \\
\hline-62.072 & -48.463 & -92.322 & -85.279 & -42.989 \\
\hline-124.01 & -129.756 & -148.149 & -123.711 & -97.887 \\
\hline 380.12 & 404.631 & 364.22 & 351.162 & 390.095 \\
\hline-294.56 & -264.788 & -319.406 & -284.408 & -290.207 \\
\hline
\end{tabular}


Figure 3. Natural logarithm of standard deviation of group estimates across periods (Experiment 1)

A: All Data Pooled

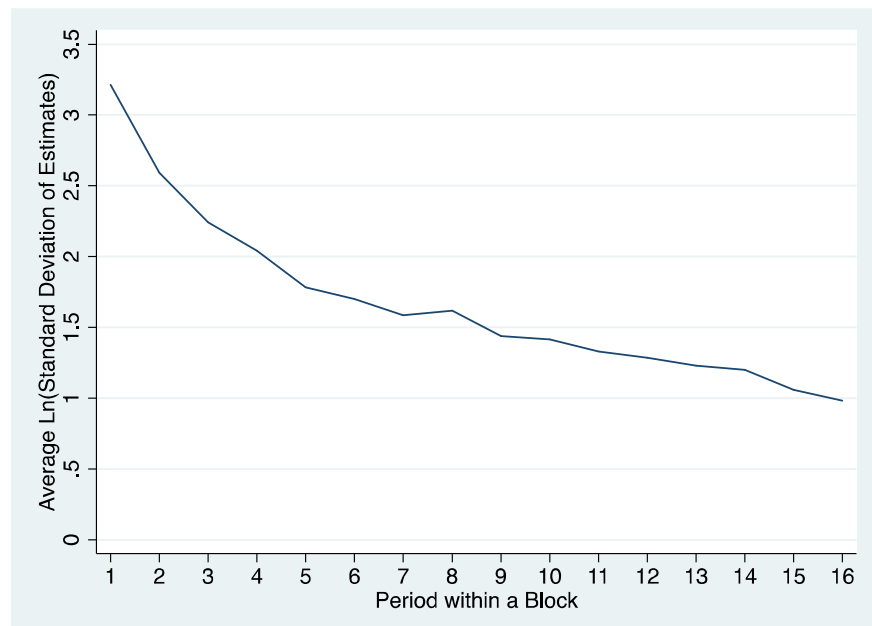

C: By Parameter Set

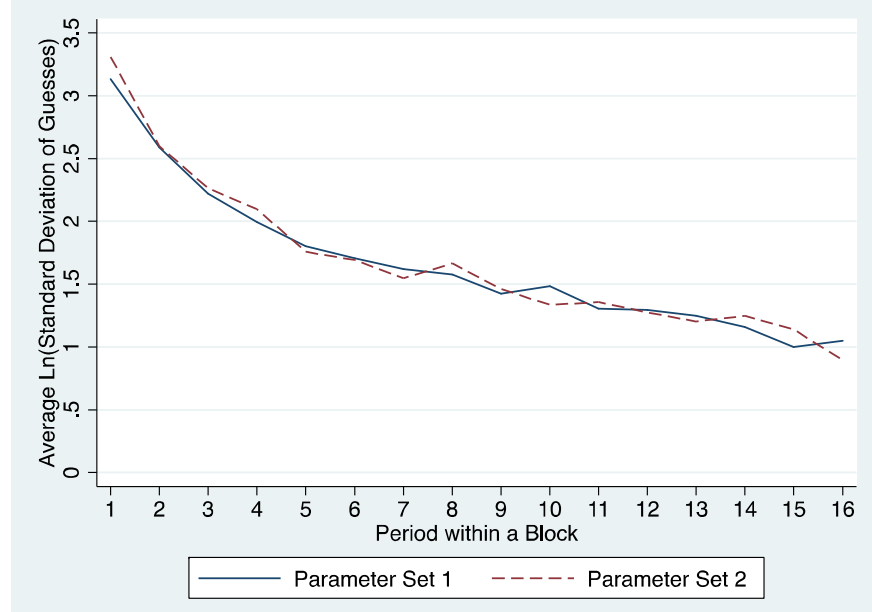

B: By Block

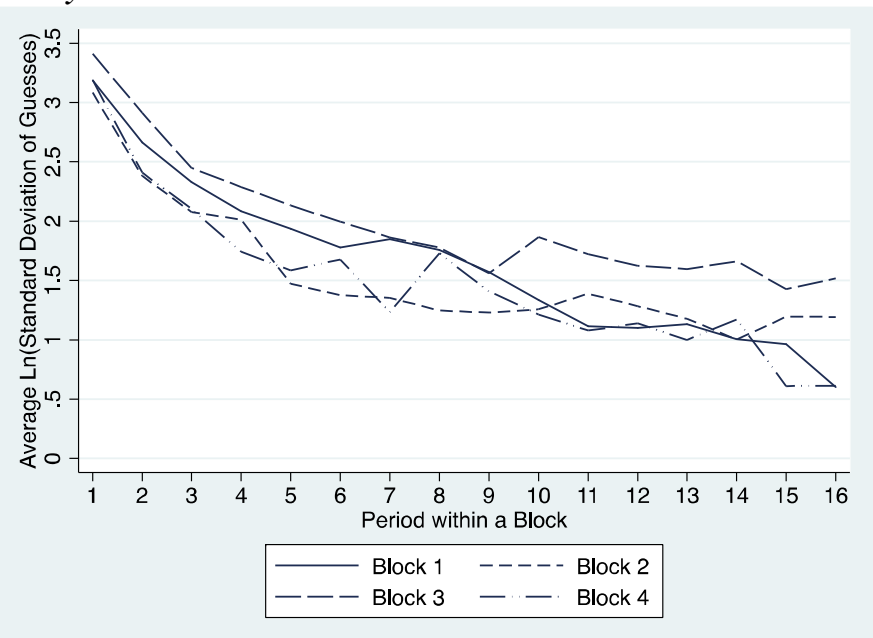

D: By Network

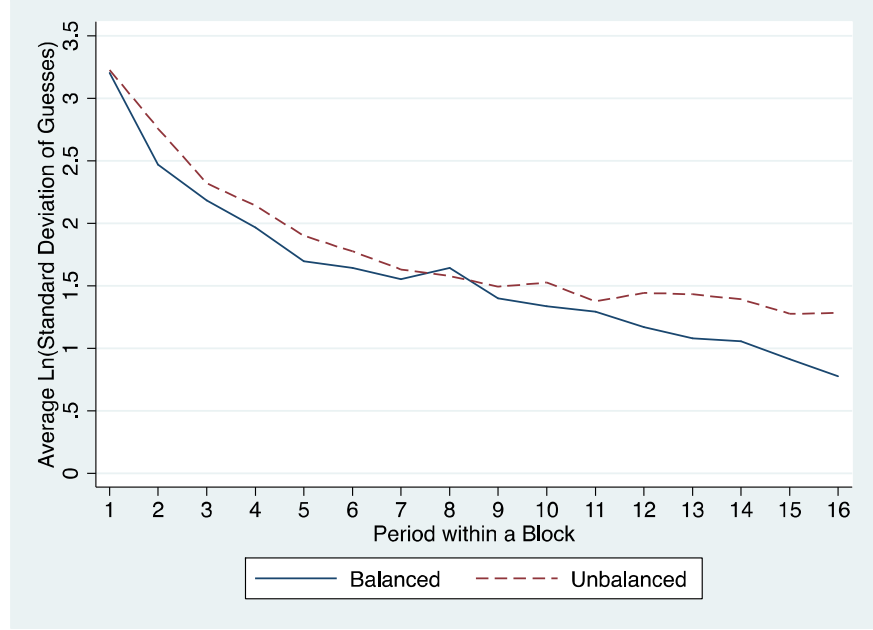


Figure 4. Proportion of groups with standard deviations of estimates below certain thresholds (Experiment 1)

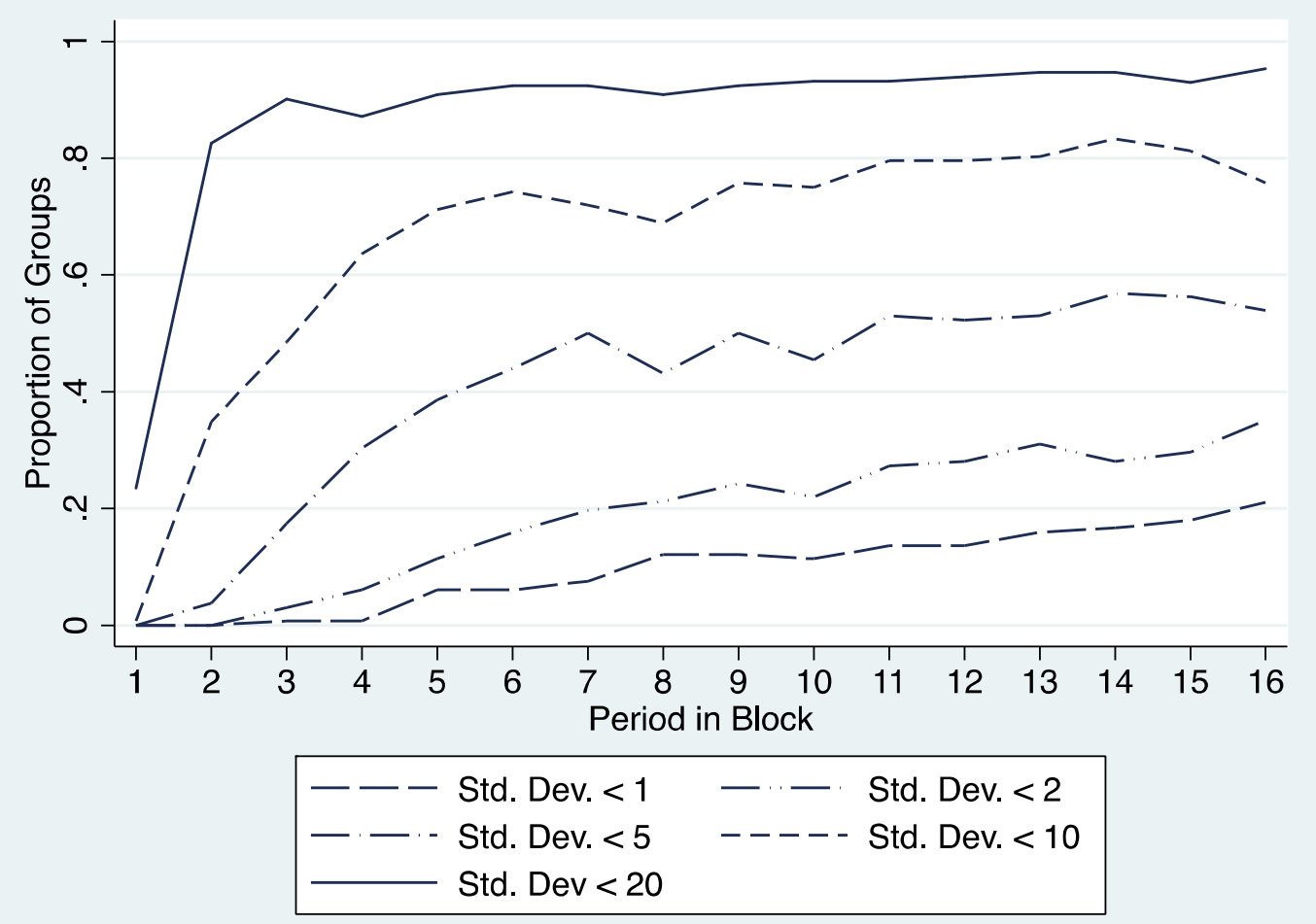


Figure 5. Median bias in mean group estimates over periods 9-16 of block (Exp. 1)

A: Parameter Set 1

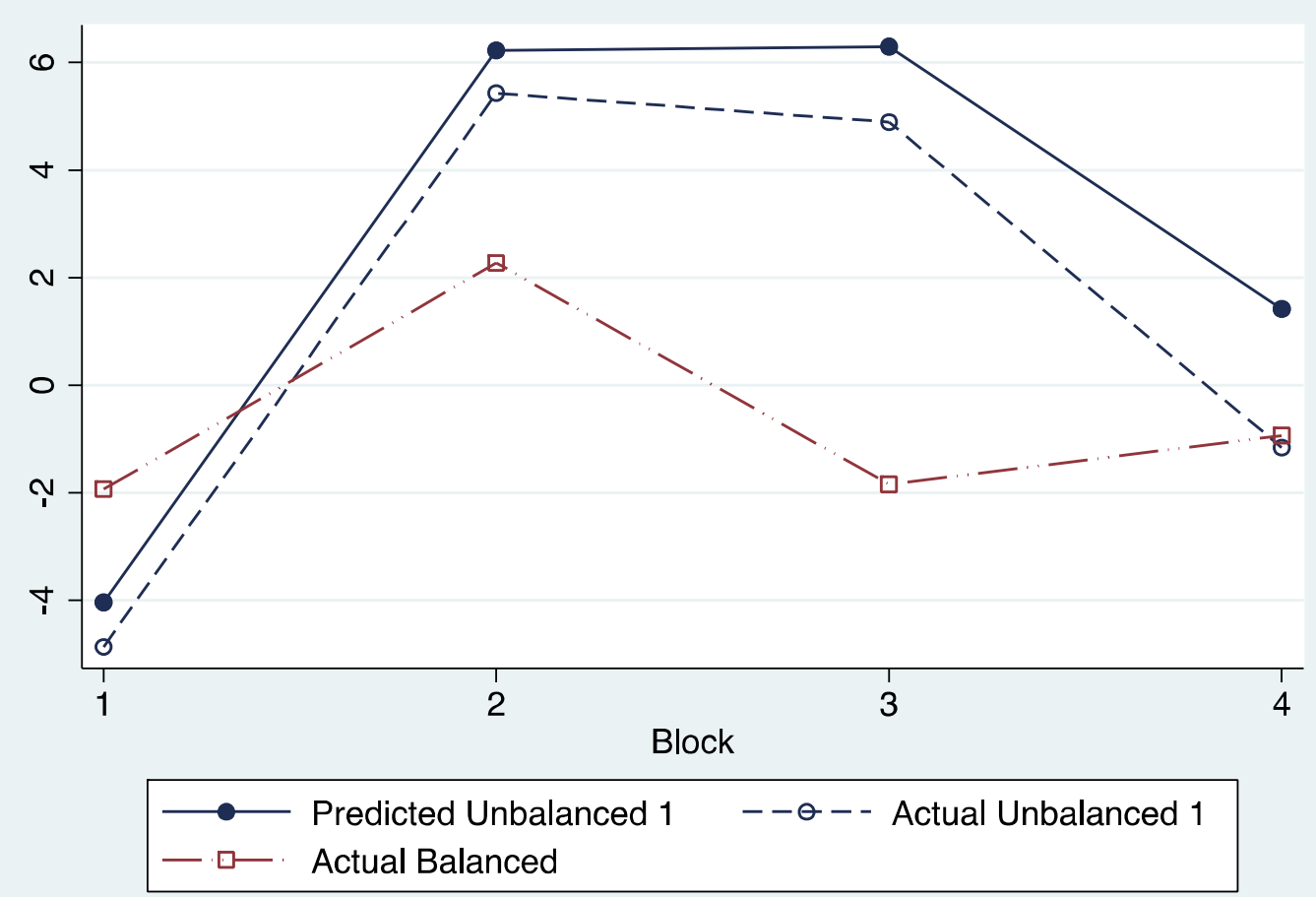

Note: Experiment 1; Parameter Set 1 only; predicted UB1 weights: 0.38, 0.07, 0.19, 0.36

B: Parameter Set 2

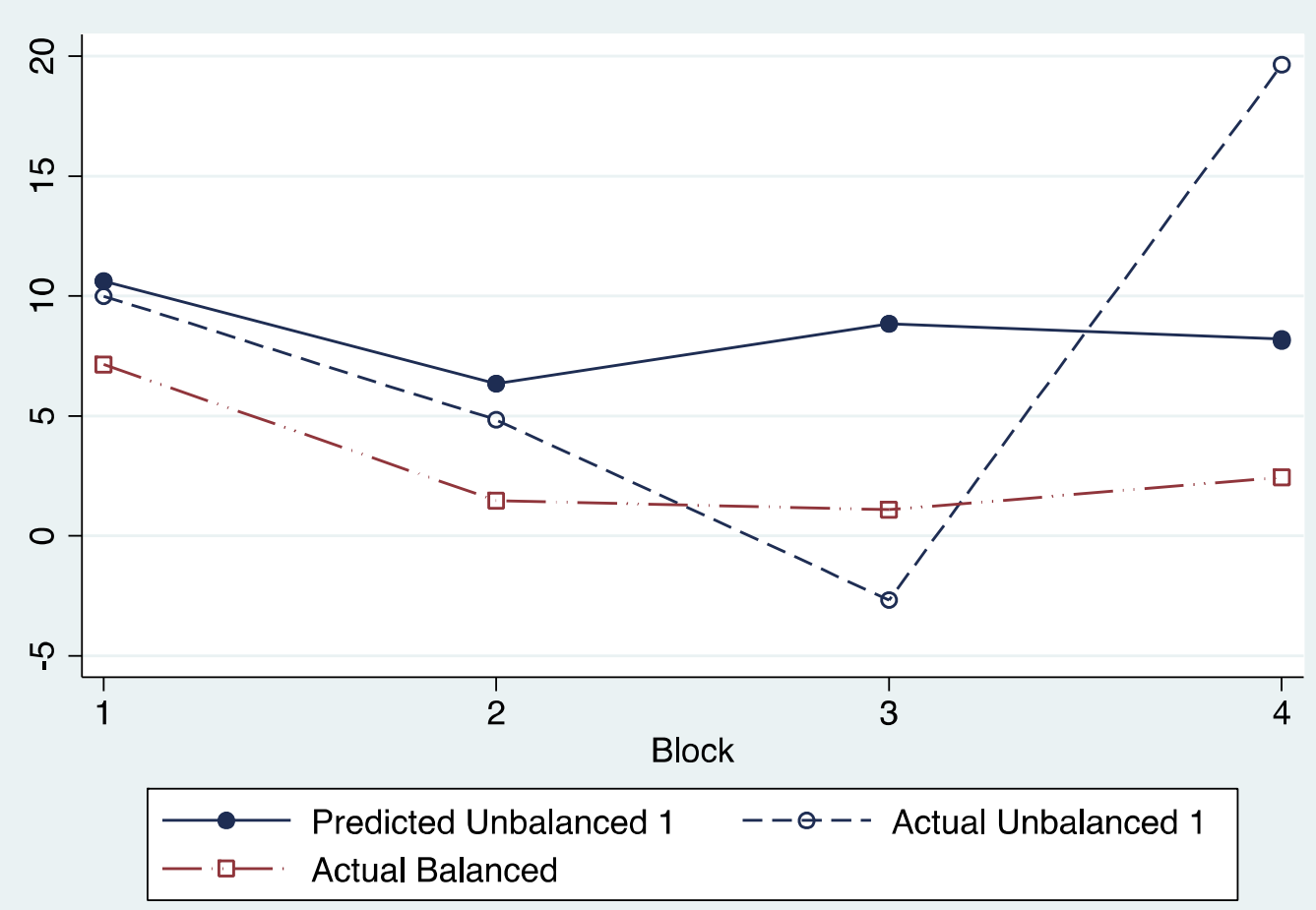

Note: Experiment 1; Parameter Set 2 only; predicted UB1 weights: 0.38, 0.07, 0.19, 0.36 
Figure 6. Natural logarithm of standard deviation of group estimates across periods (Experiment 2)

A: All Data Pooled

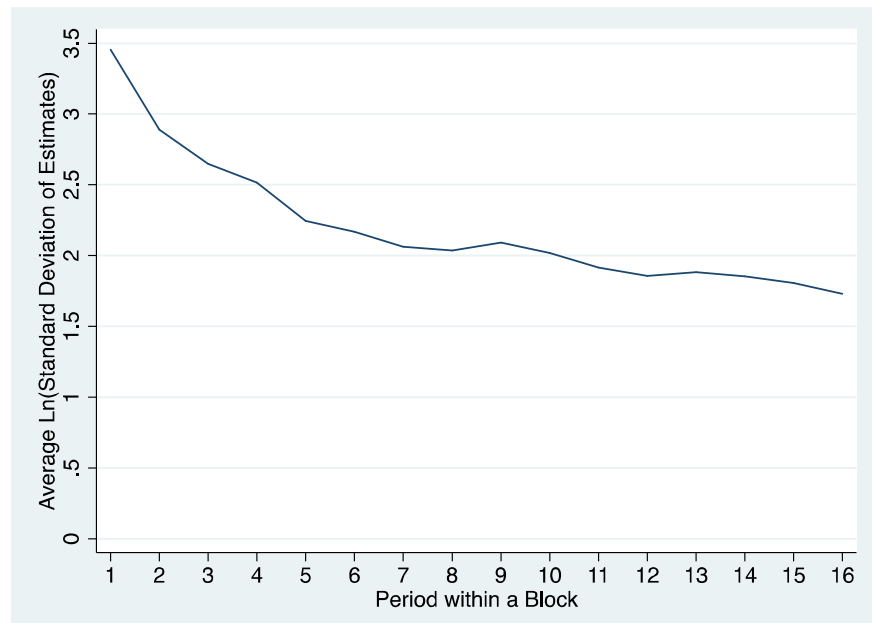

C: By Parameter Set

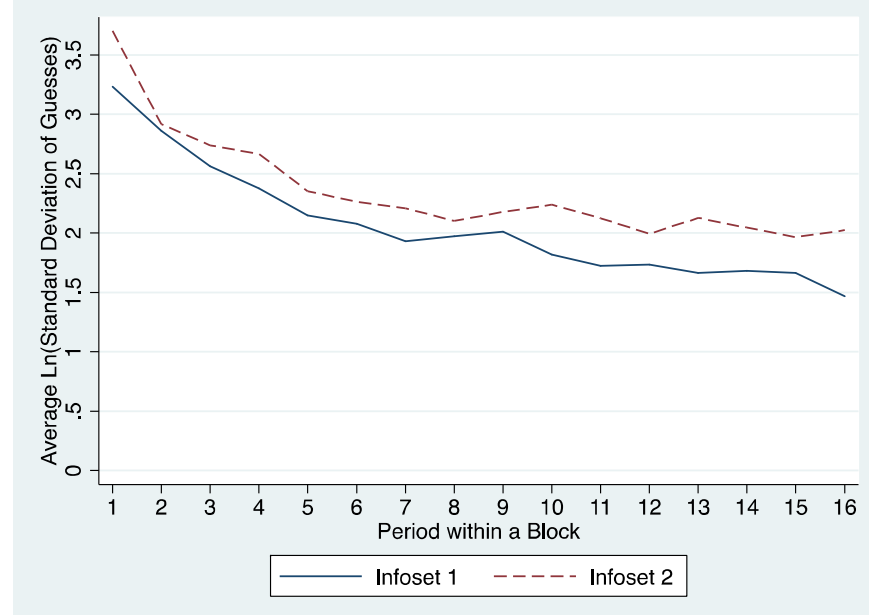

B: By Block

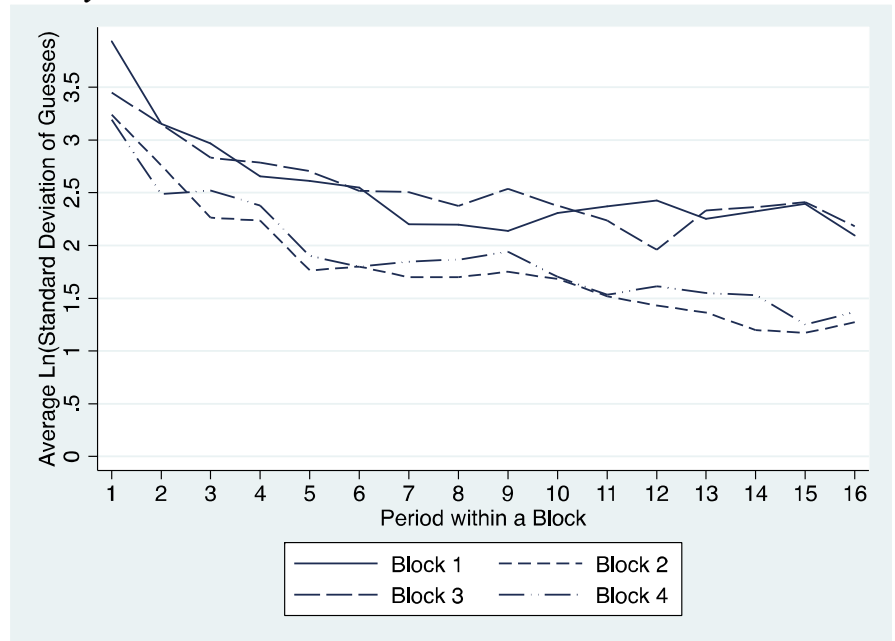


Figure 7. Proportion of groups with standard deviations of estimates below certain thresholds (Experiment 2)

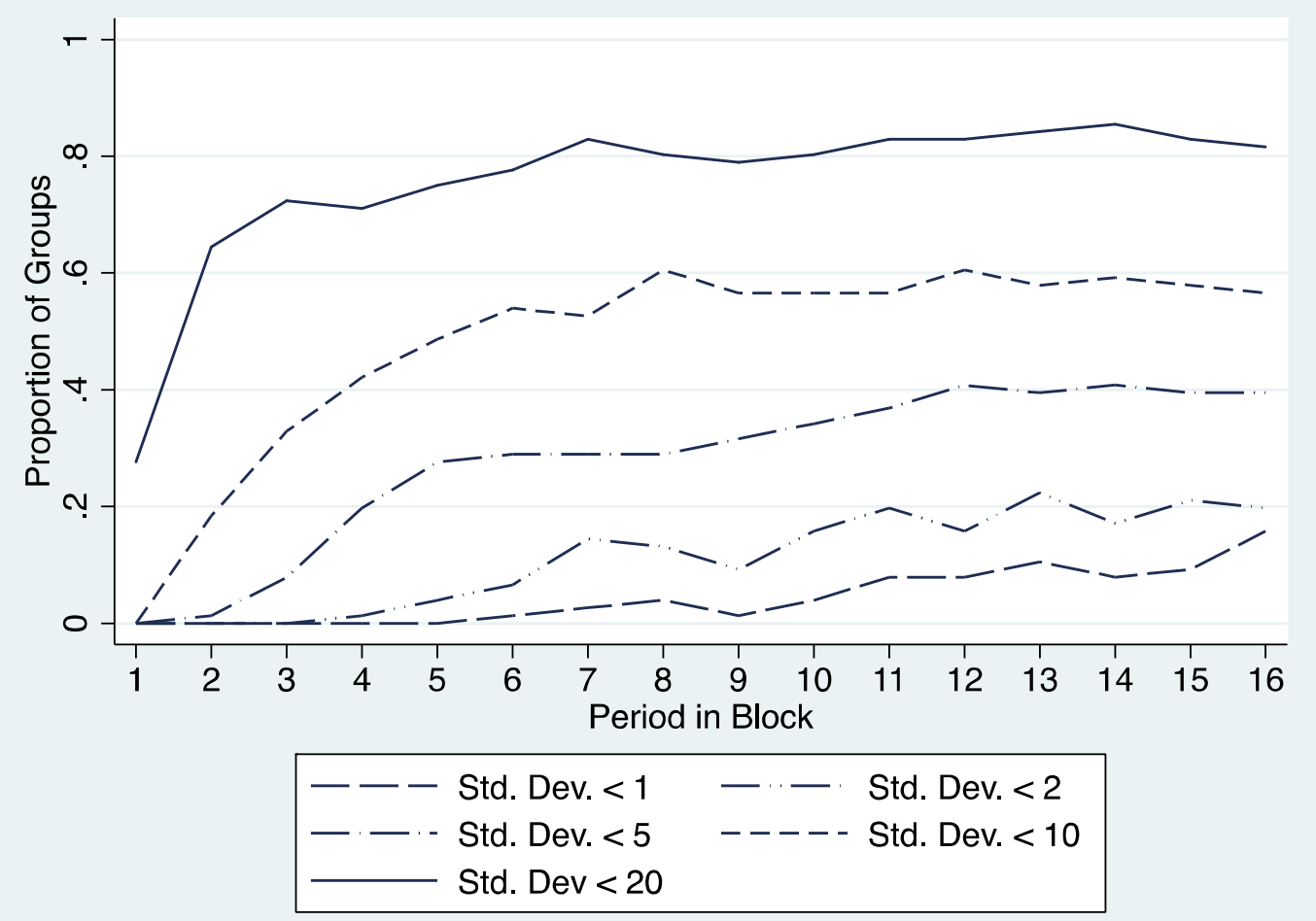

